\title{
Detection and Related Research of Serum Levels of IL-2, IL-6, IL-8 and ET in Patients with Hyperthyroidism
}

\author{
Li Hongyan ${ }^{1,2, ~ *, ~ Z h a n g ~ J i n g y u ~}{ }^{3}$, Xu Qian ${ }^{1}$, Wang Cuicui ${ }^{1}$, Cao Tingting ${ }^{1}$, Hou Zhenjiang ${ }^{2}$ \\ ${ }^{1}$ Department of Medical Technology of Cangzhou Medical College, Cangzhou, China \\ ${ }^{2}$ Cangzhou Thyroid Disease Engineering Research Center, Cangzhou, China \\ ${ }^{3}$ Department of Laboratory Diagnosis, Cangzhou Integrated of Traditional Chinese and Western Medicine Hospital of Hebei Province, \\ Cangzhou, China
}

\section{Email address: \\ czyzjyk@126.com (Li Hongyan) \\ *Corresponding author}

\section{To cite this article:}

Li Hongyan, Zhang Jingyu, Xu Qian, Wang Cuicui, Cao Tingting, Hou Zhenjiang. Detection and Related Research of Serum Levels of IL-2, IL-6, IL-8 and ET in Patients with Hyperthyroidism. Science Journal of Public Health. Vol. 8, No. 6, 2020, pp. 177-180.

doi: $10.11648 /$ j.sjph.20200806.14

Received: October 23, 2020; Accepted: November 24, 2020; Published: November 27, 2020

\begin{abstract}
Objective: To discuss the changes of serum levels of IL-2, IL-6, IL-8 and ET in patients with hyperthyroidism, and to analyze the correlation between them and thyroid function indexes, and to find more objective indexes for the diagnosis, treatment and prevention of hyperthyroidism. Methods: 251 patients with hyperthyroidism (Study Group) and 130 healthy persons (control group) were selected. Five indexes of serum thyroid function: serum free triiodothyronine (FT3), total triiodothyronine (TT3), serum free thyroxine (FT4), total thyroxine (TT4) and thyroid stimulating hormone (TSH) were measured by electrochemiluminescence. The levels of seruminterleukin-2 (IL-2), interleukin-6 (IL-6), interleukin-8 (IL-8) were detected by ELISA double antibody sandwich method, and the level of endothelin (ET) was measured by enzyme linked immunosorbent assay. Results: The level of IL-2 in study group was significantly lower $(\mathrm{P}<0.05)$, which was negatively correlated with serum FT3, FT4, TT3 and TT4; The levels of IL-6, IL-8 and ET were significantly increased (P $<0.05)$, and positively correlated with serum FT3, FT4, TT3 and TT4; The four indexes were not correlated with serum TSH. Conclusions: IL-2, IL-6, IL-8 and ET are involved in the occurrence and development of hyperthyroidism, and monitoring the changes of each index has certain clinical application value for the diagnosis, pathogenesis research, judgment and prognosis of hyperthyroidism.
\end{abstract}

Keywords: Hyperthyroidism, IL-2, IL-6, IL-8, ET

\section{Introduction}

As an endemic disease in Cangzhou City and its surrounding areas, hyperthyroidism has attracted much attention for many years. Hyperthyroidism refers to an endocrine disease caused by hyperthyroidism and (or) increased thyroid hormone level in blood circulation. However, the lesion is not limited to the thyroid gland, but a multi system syndrome. The main clinical manifestations are increased excitability of digestive, circulatory and nervous systems and hypermetabolism [1], among which cardiovascular and nervous systems are more common [2]. More than $80 \%$ of hyperthyroidism is caused by Graves' disease, which is an autoimmune disease [3]. Studies have shown that cytokines play an important role in immune regulation and immune stabilization of thyroid cells [4].

In order to find more objective indexes for the diagnosis, treatment and prevention of hyperthyroidism, the changes of serum levels of interleukin-2 (IL-2), interleukin-6 (IL-6), interleukin-8 (IL-8) and endothelin (ET) in serum of patients with hyperthyroidism and their correlation with thyroid function indexes were determined.

\section{Data and Methods}

\subsection{Included Objects}

From January 2017 to January 2019, 251 patients with hyperthyroidism (including 98 males and 153 females, aged 
21-72 years, with an average of $42.2 \pm 15.1$ years) were selected as the study group. The diagnostic criteria of hyperthyroidism were as follows: with symptoms or signs of hyperthyroidism, serum FT3 $>7.1 \mathrm{pmol} / 1, \mathrm{FT} 4>22 \mathrm{pmol} /$ $\mathrm{L}, \mathrm{TSH}<0.27 \mathrm{miu} / 1$ [5]. All cases were diagnosed as primary cases without any treatment. The endocrine system and non thyroid diseases were excluded. During the same period, 130 healthy people (58 males and 72 females, aged 20-70 years, with an average of $45.3 \pm 12.5$ years) were selected as the normal control group. The two groups were comparable in gender and age. This study was approved by the medical ethics committee of Cangzhou integrated of traditional Chinese and Western medicine Hospital of Hebei Province.

\subsection{Research Method}

After receiving a fasting hemospasia in the early morning, venous blood $(4 \mathrm{ml})$ was collected from patients and healthy persons, resting for 1 to 2 hours at room temperature and then serum was separated by high-speed centrifugation and stored in refrigerator at $-40^{\circ} \mathrm{C}$.

The five indexes of serum thyroid function: free triiodothyronine (FT3), total triiodothyronine (TT3), free thyroxine (FT4), total thyroxine (TT4) and thyroid stimulating hormone (TSH) were determined by Cobas E602 Automatic Immune Diagnostic Assay System, the reagents are all Roche's specialized supporting products. IL-2, IL-6 and IL-8 were detected by ELISA double antibody sandwich method, and the operation was strictly in accordance with the instructions. The results were reported in $\mathrm{pg} / \mathrm{ml}$. ET was determined by ELISA kit of Shanghai Kanu Biotech Co. Ltd.

\subsection{Statistical Methods}

Spss 19.0 statistical software was used. The data was expressed as $\bar{x} \pm \mathrm{S}$. The comparison of measurement data was conducted by t-test, and the correlation between various factors was analyzed by linear correlation analysis. $\mathrm{P}<0.05$ suggests that there were significant differences.

\section{Results}

\subsection{Comparison of Determination Results of Serum IL-2, $I L-6, I L-8$ and $E T$}

Compared with the normal control group, the level of IL-2 in the study group was significantly lower $(\mathrm{P}<0.05)$, and the levels of IL-6, IL- 8 and ET were significantly increased $(\mathrm{P}<0.05)$, and the difference was statistically significant $(\mathrm{P}<0.05)$ (Table 1).

Table 1. The levels of serum IL-2, IL-6, IL-8andET $(x \pm s)$.

\begin{tabular}{lll}
\hline & control group $(\mathrm{n}=\mathbf{1 3 0})$ & study group $(\mathbf{n = 2 5 1})$ \\
\hline IL-2 $(\mathrm{pg} / \mathrm{ml})$ & $476.1 \pm 35.6$ & $172.4 \pm 24.8$ \\
IL-6 $(\mathrm{pg} / \mathrm{mL})$ & $1.3 \pm 0.1$ & $2.7 \pm 0.2$ \\
IL-8 $(\mathrm{pg} / \mathrm{mL})$ & $60.4 \pm 13.7$ & $90.4 \pm 16.5$ \\
ET $(\mathrm{ng} / \mathrm{L})$ & $110.19 \pm 12.62$ & $137.28 \pm 14.57$ \\
\hline
\end{tabular}

\subsection{Correlation Analysis of Serum IL-2, IL-6, IL-8, ET and Thyroid Function Indexes}

Correlation analysis of serum IL-2, IL-6, IL-8, ET and thyroid function indexes in the study group showed that IL-2 was negatively correlated with serum FT3, FT4, TT3 and TT4, while IL-6, IL-8 and ET were positively correlated with serum FT3, FT4, TT3 and TT4, but were not correlated with serum TSH (Table 2).

Table 2. Comparison of correlation between IL-2, IL-6, IL-8, ET and thyroid function indexes in Study Group.

\begin{tabular}{llllll}
\hline & $\mathbf{F T}_{\mathbf{3}}$ & $\mathbf{F T}_{\mathbf{4}}$ & $\mathbf{T T}_{\mathbf{3}}$ & $\mathbf{T T}_{\mathbf{4}}$ & $\mathbf{T S H}$ \\
\hline \multirow{2}{*}{$\mathrm{Il}-2$} & $\mathrm{r}=-0.800$ & $\mathrm{r}=-0.612$ & $\mathrm{r}=-0.431$ & $\mathrm{r}=-0.401$ & $\mathrm{r}=0.621$ \\
& $P=0.011$ & $P=0.003$ & $P=0.012$ & $P=0.004$ & $P=0.147$ \\
Il-6 & $\mathrm{r}=0.501$ & $\mathrm{r}=0.415$ & $\mathrm{r}=0.476$ & $\mathrm{r}=0.420$ & $\mathrm{r}=0.187$ \\
& $P=0.013$ & $P=0.008$ & $P=0.023$ & $P=0.018$ & $P=0.232$ \\
Il-8 & $\mathrm{r}=0.707$ & $\mathrm{r}=0.520$ & $\mathrm{r}=0.479$ & $\mathrm{r}=0.741$ & $\mathrm{r}=0.453$ \\
& $P=0.012$ & $P=0.013$ & $P=0.002$ & $P=0.005$ & $P=0.361$ \\
ET & $\mathrm{r}=0.724$ & $\mathrm{r}=0.632$ & $\mathrm{r}=0.514$ & $\mathrm{r}=0.512$ & $\mathrm{r}=0.501$ \\
& $P=0.112$ & $P=0.006$ & $P=0.014$ & $P=0.005$ & $P=0.610$ \\
\hline
\end{tabular}

\section{Discussions}

Clinical studies have found that most of hyperthyroidism is caused by Graves' disease [6], patients with hypermetabolism, nervous, circulatory, digestive and other systems show high excitability, Without early diagnosis and timely and effective treatment, the patient's condition will further aggravate and develop into hyperthyroid heart disease, which will seriously affect the patient's health and quality of life. Although the academic community has been exploring, but the pathogenesis of hyperthyroidism has not been completely clear. As an important factor affecting cellular immune response, cytokines have been a research hotspot for many years. Studies have shown thatthe occurrence and development of hyperthyroidism are related to cytokines [7], which can affect the function of thyroid cells and promote the antigenicity of thyroid [8]. The changes of cytokine network play an important role in the occurrence, development and prognosis of hyperthyroidism.

IL-2, IL-6 and IL-8 are multifunctional cytokines produced by lymphocyte and participate in a variety of biological effects. IL-2 is mainly produced by Th1 cells under the stimulation of antigen or mitogen, and can play an immune enhancing role by promoting the differentiation of $\mathrm{T}$ cells [9]. Its level can reflect the activation degree of Th1 cells and the cellular immune function of the body to a certain extent [10, 11]. Feng Xiaoqian et al found that the levels of Th1 and IL-2 in children with Henoch Schonlein purpura, a common autoimmune disease in children, were significantly decreased [12], while the levels of Th2 and IL-4 were significantly increased, which verified the viewpoint of Th1 / Th2 imbalance [13]. Wu Huaxianginterpreted the recommendation of low-dose interleukin-2 in the treatment of Systemic Lupus Erythematosus, and found that the serum IL-2 level decreased significantly in patients with autoimmune diseases Systemic Lupus Erythematosus [14], and the number of regulatory $T$ cells (Treg) decreased, and the balance of immune tolerance 
was destroyed, which results in immune damage of their own tissues and organs of SLE patients [15].

My research found that the level of IL-2 in the study group was decreased, and negatively correlated with serum FT3, FT4, TT3 and TT4, which was consistent with the study of Geng Mingxia et al [16]. It is speculated that the reason may be that $\mathrm{T}$ cells are continuously activated in hyperthyroidism patients, while the ability of Th1 cells to produce IL-2 is decreased; the activated Th1 cells express a large number of IL-2 receptors (IL-2R) and combine with IL-2, which further reduces the IL-2 in patients. The decrease of IL-2 results in the decrease of Th1 cell's inhibitory effect on Th2 cell, which promotes the activation of $\mathrm{Th} 2$ cell, enhances the auxiliary effect on B cell, and further promotes the production of autoantibody, thus promoting the development of hyperthyroidism. The decrease of IL-2 results in the decrease of Th1 cell immune clearance ability and imbalance of immune cell network regulation, which further aggravates the immune function disorder of the body [17]. Therefore, it is reasonable to speculate that the decrease of IL-2 level may be conducive to the diagnosis of hyperthyroidism; if patients are treated with a certain dose of IL-2, the state of immune dysfunction may be gradually alleviated, but clinical research is still needed.

IL-6 is a cytokine secreted by T lymphocytes, monocyte macrophages and other cells, with multiple functions [18], which can activate and regulate immune cells and play an important role in promoting inflammatory response [19]. IL-8 is an important inflammatory mediator from multicellular sources [20]. In a variety of pathological environments, such as autoimmune diseases and metabolic diseases, IL-8 can enhance the body's inflammatory response, immune response and other processes, so as to achieve the purpose of sterilization and cell damage [17]. My research found that the levels of IL-6 and IL-8 were different from IL-2. The levels of IL-6 and IL-8 were increased in serum of patients with hyperthyroidism, which was consistent with the studies of Zhang Yunming and Wu Jiali [21, 22]. The study also found that IL-6 and IL-8 were positively correlated with serum FT3, FT4, TT3 and TT4. Thus, IL-6 and IL-8 play an important role in the pathogenesis of hyperthyroidism. It may be that IL-6 and IL-8 can activate Th2 cells and enhance their auxiliary effect on B cells, thus promoting the production of autoantibodies and accelerating the development of hyperthyroidism.

ET is a vasoactive peptide secreted by endothelial cells [23], which acts on vascular endothelium to regulate vascular tension, and plays a role in vascular damage by promoting endothelial cell apoptosis and leukocyte adhesion [24], which can reflect the degree of vascular endothelial damage [25]. My research found that the level of ET in the study group was significantly increased and positively correlated with serum FT3, FT4, TT3, TT4, which indicated that the increase of thyroid hormone damaged the vascular wall, resulting in the increase of ET synthesis or release by vascular endothelial cells. Therefore, in the process of treatment, timely monitoring of ET level may have certain reference value for observing whether the treatment is effective, whether the hemodynamics of patients is restored to normal, and to prevent or reduce cardiovascular damage in patients with hyperthyroidism.

\section{Conclusion}

To sum up, IL-2, IL-6, IL-8 and ET are involved in the occurrence and development of hyperthyroidism, and monitoring the changes of each index has certain clinical application value for the diagnosis, pathogenesis research, judgment and prognosis of hyperthyroidism.

\section{Acknowledgements}

Self-financed Project of Hebei Science and Technology Research and Development Program (No. 162777205), Cangzhou Science and Technology Research and Development Guidance Program Project (Ref. 151302011).

\section{References}

[1] Hou Zhen-jiang, Zhang Jing-yu, Bian Yun-zhuo, et al. Changes of serum lipid level, its ratio and cystatin $\mathrm{C}$ level in patients with hyperthyroidism and subclinical hyperthyroidism [J]. Shandong Medical Journal, 2017, 57 (39): 91-93.

[2] Li Mingxiu, pan Liangming, Jian Shucai, et al. Relationship between urinary protein level and thyroid hormone level in patients with hyperthyroidism [J]. Journal of Chengdu Medical College, 2016, 11 (2): 232-234.

[3] Pang Aiping. Clinical significance of characteristics and related biochemical indexes in patients with Graves disease complicated with hyperthyroid heart disease [D]. Guangxi Medical University, 2018.

[4] Zhang Buwen, Feng Yunfei. Expression of serum IL-3, IL-2 and SIL-2R in patients with Graves disease [J]. Modern Practical Medicine, 2014, 26 (04): 423-425.

[5] Shan Zhongyan. Guide to the diagnosis and treatment of thyroid diseases in China [J]. Chinese Journal of Practical Internal Medicine, 2008, 28 (4): 260-261.

[6] Kan Guangdi, Yi Fangliang. Xiakucao Granules Combined with methimazole tablets in the treatment of hyperthyroidism and its effect on thyroid hormone, bone metabolism indexes and immune related cytokines [J]. Hebei Traditional Chinese medicine, 2020, 42 (06): 892-895 + 901 .

[7] Zhang Yun-ming, Song Ming-qiang. Changes of IL-2, IL-6, TNF concentration after 131I therapy in patients with hyperthyroidism from Graves' disease [J]. Guide to Chinese Medicine, 2013, 11 (2): 8-9.

[8] Wu Tengfei, Zeng Juanhua, Cong Yilei, et al. Effect of Yingliu mixture combined with methimazole on thyroid function and related cytokines in patients with Graves disease [J]. Journal of Shanghai University of traditional Chinese medicine, 2016, 30 (1): $19-22$.

[9] Batista-Duharte A, Téllez-Martínez D, Fuentes D, et al. Molecular adjuvants that modulate regulator T cell function in vaccination: A critical appraisal [J]. Pharmacol Res, 2018, 129: 237-250. 
[10] Lee JJ, Escher JC, Shuman MJ, et al. Final adult height of children with inflammatory bowel disease is predicted by parental height and patient minimum height Z-score. Inflammatory Bowel Diseases, 2010, 16 (10): 1669-1677.

[11] Ouyang Jun. correlation between interleukin-2 and interleukin-37 in vaginal lavage fluid and recurrence of senile vaginitis [J]. Chinese Journal of Gerontology, 2020, 40 (13): $2821-2823$

[12] Feng Xiaoqian, TIan Xiuying, Luo Yarui. The relationships of henoch-schonlein purpura to $\mathrm{T}$ cell subsets and effector cytokines [J/OL]. Chongqing Medical Journal: 1-9.

[13] Li Y, Zhou Y, Zhu D, et al. The role of $\mathrm{T}$ cells in the development of Henoch-Schonlein purpura [J]. Front Biosci (Landmark Ed), 2018, 23: 837-851.

[14] Wu Huaxiang. The recommendation of low-dose interleukin-2 in the treatment of Systemic Lupus Erythematosus [J]. Zhejiang medical journal, 2020, 42 (16): 1681-1683.

[15] Zhu Y, Huang Y, Ming B, et al. Regulatory T-cell levels in systemic lupus erythematosus patients: a meta-analysis [J]. Lupus, 2019, 28 (4): 445-454. DOI: $10.1177 / 0961203319828530$.

[16] Geng Mingxia, Ma Jie, He Fengrong, et al. Detection and analysis of serum cytokine levels and thyroid function indexes in patients with Graves disease [J]. Journal of microcirculation, 2005 (03): $27-28+1-2$.

[17] Lin Shuzhou, Cai Wen. The significance of serum IL-2, IL-6 and IL-8 in the early diagnosis of Crohn's disease [J]. China Practical Medicine, 2013, 8 (16): 55-56.

[18] Tang Qing, Li Song, Zhong Yuanfeng, Li Chen, et al. The value of serum HDL-C combined with PCT, CRP, IL-6 in the evaluation of the severity of abdominal infection [J]. Laboratory Medicine and Clinical, 2020, 17 (13): 1839-1842 + 1846.

[19] Huang Limin. Effect of xueshuantongmai Decoction on clinical efficacy and IL-6 level in patients with cerebral thrombosis [J]. Contemporary Medicine, 2020, 26 (20): 46-48.
[20] Du Mengwei, Bai Xue, Li Jiaqi, et al. Interleukin-8 and tumor immune escape $[\mathrm{J} / \mathrm{OL}]$. Chinese Bulletin of Life Sciences, 2020 (06): 1-11 [2020-07-21] https://doi.org/10.13376/j.cbls/2020065.

[21] Zhang Yunming, Song Mingqiang. Effects of 131I treatment on IL-2, IL-6 and TNF - $\alpha$ cytokines in Graves' disease [J]. Chinese medical guide, 2013, 11 (2): 8-9.

[22] Wu Jiali, Zheng Yingying, Ma Qiling. Study on thechanges of IGF-1 and IL-8 in the Serum of the Hyperthyroidism Patients [J]. Guizhou Medical Journal, 2010, 34 (03): 224-225.

[23] Chai Kuolu, Tan Liyan, Yang Yuhong, et al. Changes and clinical significance of plasma endothelin and nitric oxide in patients with hyperthyroidism [J]. Heilongjiang Medicine And Pharmacy, 2004 (04): 34-35.

[24] Zhao song, Cui Yingying, Yan Jinju, et al. Diagnostic value of serum Hcy, ET-1 combined with UACR for early renal injury in patients with preeclampsia $[\mathrm{J}]$. International Journal of Laboratory Medicine, 2020, 41 (14): 1685-1689.

[25] Xu Ziwen. Effect of Xinhuosu on vascular endothelial function and inflammatory factors in patients with heart failure after acute myocardial infarction [J]. Contemporary Medicine, 2020, 26 (22): $175-176$

\section{Biography}

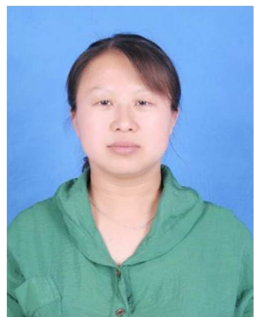

Li Hongyan (1976.8-), female, associate professor, chief technician, master of medicine, member of Cangzhou Thyroid Disease Engineering Research Center, research direction: clinical laboratory diagnostics. 